\title{
Recoverable catalysts. Ultimate goals, criteria of evaluation, and the green chemistry interface*
}

\author{
J. A. Gladysz \\ Institut für Organische Chemie, Friedrich-Alexander Universität Erlangen-Nürnberg, \\ Henkestrasse 42, 91054 Erlangen, Germany
}

\begin{abstract}
Attempts are made to define the "ideal synthesis", "ideal catalyst", and "ideal recoverable catalyst". Although these unattainable limits can never be realized, they help to focus attention on attributes chemists should strive for. Criteria for evaluating catalyst recovery are analyzed, and increased attention to the critical and constructive evaluation of competing "green" chemical technologies is advocated.
\end{abstract}

\section{INTRODUCTION}

This issue of Pure and Applied Chemistry features papers related to a number of presentations at the CHEMRAWN XIV conference in Boulder, Colorado in June, 2001. Collectively, the articles illustrate the very broad range of important issues addressed. One area identified for future emphasis is the development of standards for quality research in green or sustainable chemistry. Admittedly, any judgment of research quality carries a subjective component. However, a constructive discussion or debate serves to sharpen and focus consensus. For example, within an established field such as organic chemistry, there is virtually unanimous agreement on what constitutes adequate characterization of a new compound, or a structure proof. Within the younger field of green chemistry, and its many diverse facets, standards are still evolving.

In judging quality, it is certainly possible for a given scientific community to become hypercritical. However, it is also important to avoid uncritical and semi-evangelical love-fests. Various subdisciplines of organic chemistry have periodically probed the former limit, testing the boundaries of civil debate. Mechanistic investigations frequently prompt emotional discussions, and anyone familiar with studies of reactive carbocation intermediates during the 1950s and 1960s can enumerate many specific examples. However, negativism has an essential role in the scientific process. As repeatedly emphasized by many textbooks and scientists, it is impossible to "prove" a mechanism. Rather, one proposes a model, and tries to disprove it through experiment. Five different chemists might favor five different mechanisms. The intellectual and experimental challenge is then to design a series of tests that will exclude or support the competing possibilities. In order words, the task at hand is intrinsically adversarial - to prove something wrong or inconsistent with known facts.

From a humanistic perspective, such negativism can be harsh and is probably not the best way to recruit students to a field. When the lead-off question is "what is wrong with this picture?", it is no wonder that some will be driven to "softer" disciplines where questions of the type "what is pleasing to the eye in this painting?" or "what did you like about this book?" represent the norm. However, I for one will resist positioning green chemistry as a "softer" form of chemistry. I am furthermore convinced that such discussions and analyses constitute the best way to train students and educate the next generation

\footnotetext{
*Adapted from a lecture presented at the IUPAC CHEMRAWN XIV Conference on Green Chemistry: Toward Environmentally Benign Processes and Products, Boulder, Colorado, USA, 9-13 June 2001. Other presentations are published in this issue, pp. 1229-1330.
} 
of researchers. These debates represent the sharpening-stones that help prepare everyone for future urgent scientific problems.

Green chemistry, like organic chemistry, offers abundant potential for constructive analysis, debate, and criticism. New synthesis methods are constantly being developed with the idea of improving or replacing older non- or less-green technologies. There are obvious questions such as: (a) Do the new methods constitute genuine improvements? (b) Which of several competing new technologies is the best, or how do the trade-offs compare? (c) Which evaluation criteria are most important? Related issues, common to the mechanistic studies described above, include: (a) Are the experimental methods rigorous? (b) Are the conclusions rigorously supported by the data? I find the absence of attention to such questions in some forums for green chemistry to be disturbing, and a potential impediment to attracting top researchers to the field. As with most areas of science, there are lobbies within the green chemistry community. Any suppression of debate, either actively or passively (e.g., the elimination of discussion time after oral presentations), will help preserve the agenda of the more entrenched lobbies.

My oral presentation at this meeting described new recoverable fluorous catalysts [1]. Fluorous catalysis is a technique for conducting reactions under one-phase homogeneous conditions, with catalyst/product separation under two-phase conditions-commonly organic liquid/fluorous liquid [2-4]. Fluorous solvents are nontoxic and environmentally benign, but costly and persistent [3]. The new results from my coworkers show how the need for a fluorous solvent can be eliminated. Since much of our data has been reported in the primary literature [1,5], this written contribution will be cast as an analysis of general issues in recoverable catalysts, in the framework of the points raised above. In other words, how should the relative merits of our and other fluorous and nonfluorous protocols be evaluated, and what experimental ideals should be striven for?

\section{DISCUSSION}

\section{Ideal synthesis}

I begin with the so-called "ideal synthesis" or "ideal reaction", which is defined in some books devoted to strategy and design in organic synthesis [6]. It is important to note at the outset that process engineers and economists have altogether different perspectives on many points (e.g., temperature [7]), as well as additional considerations (e.g., cost trade-offs). Within the culture of synthetic chemists, this virtually unattainable limit features

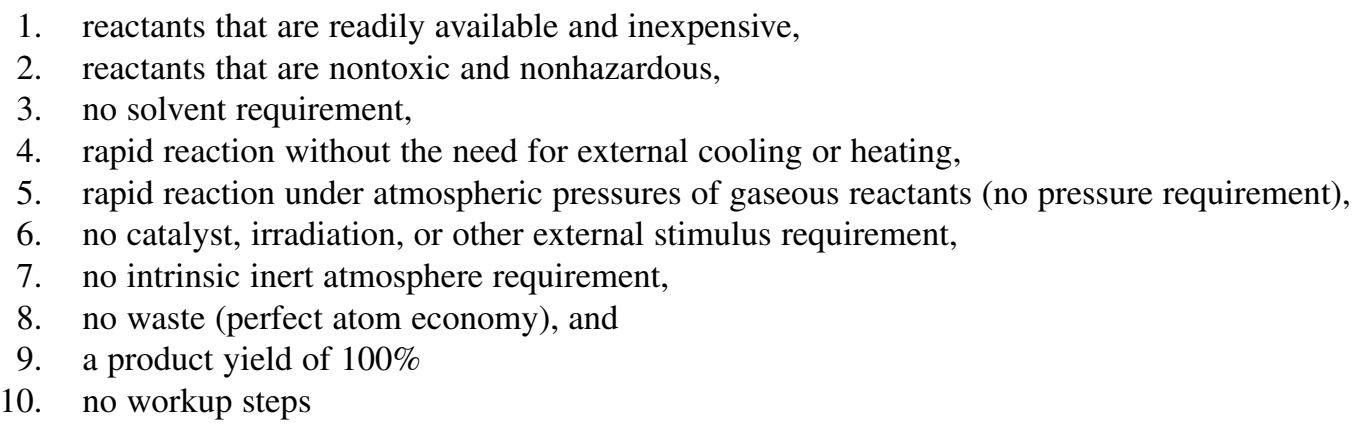

Although such reactions can be identified, they are very rare. Furthermore, they are applicable only to an infinitesimal fraction of real-world synthetic problems. However, the point to be made is that reactions that require no catalyst are preferable to those that do. Catalysis does have widely recognized benefits - thermodynamically favorable reactions that have no low-energy kinetic pathways can be rendered possible under mild conditions. Thus, once catalysts are permitted, possibilities for "ideal syntheses" increase by orders of magnitude. Even so, the number of catalyzed reactions that fulfill requirements $1-5$ and $7-10$ above is still miniscule. 


\section{Ideal catalyst}

I now relax the requirement for an "ideal synthesis", and focus strictly on catalysis of a given reaction viewed as desirable (for which there may be a co-product, an educt that poses toxicity or handling problems, or workup issues). I propose that an ideal catalyst is one that

1. produces an infinite amount of product from a single catalyst molecule (or heterogeneous equivalent), a criterion that implies

a. no deactivation under reaction conditions and

b. no poisoning under reaction conditions;

2. effects a rapid reaction (high turnover frequency or TOF) without the need for external cooling or heating;

3. effects a rapid reaction under atmospheric pressures of gaseous reactants;

4. has no intrinsic inert atmosphere requirement;

5. is insensitive to reactant impurities; and

6. gives a product yield of $100 \%$.

The first requirement is the most important for the present discussion, and implies a turnover number (TON) of infinity. Certain omissions from the above list can be questioned. For example, a catalyst that

7. is readily available and inexpensive,

8. is nontoxic and nonhazardous, and

9. requires no activation or initiation step

is obviously preferable. However, since the "infinite TON" limit is under discussion, requirements 7 and 8 asymptotically approach being negligible (zero). In real-life process chemistry, they are not. Requirement 9 would imply a "living" catalyst that directly reacts with educts.

In the "infinite TON" limit, recoverability is no longer an issue. Hence, one "queen bee" catalyst molecule (or heterogeneous equivalent) suffices. In practice, many engineering problems can be envisioned. For example, a batch process would be limited by the size of the reactor. A continuous process must somehow retain the one catalyst molecule.

Engineering aside, a surprising consequence that runs against some current thinking is obvious. Namely, the pursuit of higher-turnover-number catalysts represents a more important research direction than recoverable catalysts. A catalyst that produces 10000000 product molecules is clearly superior to one that gives 10000 product molecules and can through some protocol be recycled 1000 times. To my knowledge, the design and synthesis of catalysts with enhanced lifetimes/activities is generally not included under the rubric of green chemistry. This analysis demonstrates a fundamental and intrinsic relationship.

Nonetheless, everyone would agree that an "ideal catalyst" that is recoverable in single-molecule form is preferable to one that is eventually lost in a product stream. Thus, recoverable catalysts are a very important field for research. The relative merits of various strategies can be expected to depend upon the reaction under consideration, the reaction scale, and engineering requirements. A lively debate would ensue in nearly every case, and these value judgments are beyond the scope of this article. Rather, the remaining discussion focuses on key considerations common to all recycling protocols.

\section{Recoverable catalysts: What does recycling really mean?}

At the beginning of a reaction, one adds a catalyst precursor. Sometimes there is an induction period. This indicates some fundamental, generally irreversible change before catalysis can begin. Alternatively, NMR might show that an amine catalyst for the acylation of a nucleophile exists only 
("rests") in quaternized form under the reaction conditions. Regardless, what one is recycling is always the catalyst rest state. The rest state might also be altered during recycling (perhaps a product molecule dissociates as product is being removed). Thus, any special property being exploited for catalyst recovery must be associated with the rest state(s).

\section{Ideal recoverable catalyst}

From the above precepts, the following definition is apparent:

An "ideal recoverable catalyst" is one that can be recovered quantitatively (e.g., every molecule for a homogeneous species), either as the catalyst precursor or a functionally equivalent rest state. The other attributes of the ideal catalyst remain-particularly the high TON and TOF values.

Given how obvious the first sentence of the preceding paragraph seems, it is surprising that so little academic research on recoverable catalysts is evaluated by this criterion. However, it is often difficult to analyze and quantify catalyst recovery. The most common yardsticks are summarized in the following section.

It is possible to propose additional criteria for an ideal recoverable catalyst, and/or real-world systems that do not approach the "single molecule" limit of section on ideal catalyst. For example, a catalyst precursor that must undergo an irreversible waste-generating step (e.g., ligand dissociation) is not as desirable as one that does not. This reduces to a design exercise-i.e., how to most directly enter a catalytic cycle. A related issue, elegantly illustrated in two recent papers, is the efficiency of initiation [8]. In other words, does the entire quantity of catalyst precursor participate in catalysis, or just a small fraction?

On another level, selected generalizations on recycling methods could be considered. A catalyst that can be $100 \%$ recovered without recourse to a second liquid phase might be viewed as superior to one that can be $100 \%$ recovered via a fluorous or ionic liquid phase. However, as noted above, these represent value judgments beyond the scope of this article.

\section{Quantitative evaluation of recycling methods}

\section{Product yield as a function of cycle}

Almost without exception, papers describing catalyst recycling report these values, and/or closely related TON data. This is probably because many beginning researchers in catalysis are under the impression that "here is where the story is", or that yields are a good criterion of recoverability. Nothing could be further from the truth. Suppose an arbitrary decision has been made to let each cycle run one hour. However, under the conditions of cycle 1 product formation is in fact complete after one minute. Suppose that half the catalyst is lost in the first recycling operation. Cycle 2 could still give complete product formation in two minutes (given reasonable rate law assumptions, etc.). Suppose half the catalyst is lost on each succeeding cycle. No major yield deterioration would be noted until cycle 8 ! However, the rate of product formation or turnover frequency (TOF) would decrease markedly from cycle to cycle.

\section{TOF as a function of cycle}

This is a very meaningful measurement, but absent in the majority of papers involving catalyst recycling (including some of mine). Rate constants or conversion vs. time plots that are constant as a function of cycle represent good evidence for efficient catalyst recovery. With systems that give induction periods, comparisons between the second and third (and subsequent) cycles are more meaningful than between the first and second cycles.

\section{Catalyst recovery}

High-quality studies will seek a direct measure of catalyst recovery. This can be experimentally demanding, as very small quantities are commonly involved. Also, since the catalyst rest state is being 
recovered, the spectroscopic properties may not match those of the well-characterized catalyst precursor. Atomic absorption methods are frequently used, which introduce other assumptions and approximations (e.g., does the total metal content reflect the total catalyst content?).

\section{Catalyst decomposition and/or leaching}

High-quality studies will seek a complete mass balance for the catalyst precursor. In addition to recovered catalyst (previous point), three other quantities may be defined: a) the amount of active catalyst that is leached during reaction or recycling (note that each species on the catalytic cycle is an independent candidate for leaching); b) the amount of decomposed inactive catalyst that is leached during reaction or recycling; c) the amount of decomposed inactive catalyst that is recycled. Depending upon quantities and conditions, it may not be practical to measure all of these. However, as large families of recoverable catalysts are developed, which is an inevitable eventual outgrowth of combinatorial chemistry, these represent the obvious criteria by which recyclability will be judged. The development of suitably sensitive analytical methods therefore constitutes another research priority.

\section{SUMMARY}

In the preceding analysis, I have attempted to bring increased systematization to the evaluation of a number of issues to which subjective or ill-defined criteria are often applied. The suggestions in the section Quantitative Evaluation of Recycling Methods for comparing recoverable catalysts will in turn allow a sharper focus on the underlying green, economic, and engineering issues. The competition of various catalyst recycling techniques is healthy, and there is everything to be gained by clearly framing every problem so that ingenious new solutions can be developed.

Of course, many points in the preceding analysis represent my own subjective judgments. The viewpoints of other constituencies, such as an economist who might take as a starting point "my ideal catalyst is one that turns lead into gold", deserve to be better voiced. I welcome all return criticisms, as an ongoing debate can only lead to better versions of this document which will be of greater use to the community. Toward this goal, I am editing a special issue of Chemical Reviews on "Recoverable Catalysis" to be published in October of 2002. An amended and updated version of the above Discussion section, reflecting input received as of 1 July 2002, will appear in this volume in some form.

\section{ACKNOWLEDGMENTS}

I thank the Fonds der Chemischen Industrie (FCI) for support, and Dr. W. P. Mul (Shell Research and Technology Centre, Amsterdam), Dr. W. Tam (DuPont Central Research and Development, Wilmington), and Dr. G. Kiss (Exxon/Mobil, Clinton) for helpful comments and discussions.

\section{REFERENCES AND NOTES}

1. a) M. Wende, R. Meier, J. A. Gladysz. J. Am. Chem. Soc. 123 (2001) In press; b) C. Rocaboy and J. A. Gladysz. Manuscript in preparation.

2. a) I. T. Horváth and J. Rábai. Science 266, 72 (1994); b) I. T. Horváth. Acc. Chem. Res. 31, 641 (1998).

3. Survey of practical considerations and underlying physical principles: L. P. Barthel-Rosa and J. A. Gladysz. Coord. Chem. Rev. 578, 190-192 (1999).

4. Review literature since 1999: a) E. de Wolf, G. van Koten, B.-J. Deelman. Chem. Soc. Rev. 28, 37 (1999); b) R. H. Fish. Chem. Eur. J. 5, 1677 (1999); c) M. Cavazzini, F. Montanari, G. Pozzi, S. Quici. J. Fluorine Chem. 94, 183 (1999); d) U. Diederichsen. Nachr. Chem. Tech. Lab. 47, 805 (1999); e) E. G. Hope and A. M. Stuart. J. Fluorine Chem. 100, 75 (1999); f) E. G. Hope and A. M. Stuart. In Advanced Inorganic Fluorides, T. Nakajima, B. Îemva, A. Tressaud (Eds.), Ch. 13, 
p. 403, Elsevier, Amsterdam (2000); g) A. Endres and G. Maas. Chemie Unserer Zeit 34, 382 (2000).

5. a) J. J. J. Juliette, D. Rutherford, I. T. Horváth, J. A. Gladysz. J. Am. Chem. Soc. 121, 2696 (1999); b) D. Rutherford, J. J. J. Juliette, C. Rocaboy, I. T. Horváth, J. A. Gladysz. Catalysis Today 42 , 381 (1998); c) L. J. Alvey, D. Rutherford, J. J. J. Juliette, J. A. Gladysz. J. Org. Chem. 63, 6302 (1998); d) M. A. Guillevic, C. Rocaboy, A. M. Arif, I. T. Horváth, J. A. Gladysz. Organometallics 17, 707 (1998); e) A. Klose and J. A. Gladysz. Tetrahedron: Asymmetry 10, 2665 (1999); f) L. V. Dinh and J. A. Gladysz. Tetrahedron Lett. 40, 8995 (1999); (g) C. Rocaboy, D. Rutherford, B. L. Bennett, J. A. Gladysz. J. Phys. Org. Chem. 13, 596 (2000); h) C. Rocaboy, W. Bauer, J. A. Gladysz. Eur. J. Org. Chem. 2621 (2000); i) L. J. Alvey, R. Meier, T. Soós, P. Bernatis, J. A. Gladysz. Eur. J. Inorg. Chem. 1975 (2000); j) T. Soós, B. L. Bennett, D. Rutherford, L. P. BarthelRosa, J. A. Gladysz. Organometallics 20, 3079 (2000).

6. My definition represents an expanded version of one given by P. A. Wender, Chem. Rev. 96, 1 (1996). "An ideal (the ultimate practical) synthesis is generally regarded as one in which the target molecule (natural or designed) is prepared from readily available, inexpensive starting materials in one simple, safe, environmentally acceptable, and resource-effective operation that proceeds quickly and in quantitative yield".

7. For example, a reaction that must be heated does not generally present an engineering or cost disadvantage. On the other hand, a reaction that requires more cooling than can be provided with normal cooling water is expensive to scale up. It is always desirable to use the heat generated in a reaction for a productive purpose, thereby indirectly reducing $\mathrm{CO}_{2}$ emissions. In many processes, inert atmosphere conditions are maintained as a matter of course, in part due to safety considerations.

8. a) M. S. Sanford, J. A. Love, R. H. Grubbs. J. Am. Chem. Soc. 123, 6543 (2001); b) A. Börner and D. Heller. Tetrahedron Lett. 42, 223 (2001). 